Revue d'histoire de l'Amérique française

DQ REVUE D.HISTOIRE DE L'AMÉRIQUE FRANÇAISE

\title{
Commerce, crédit et transactions foncières : pratiques de la communauté marchande du bourg de l'Assomption, 1748-1791
}

\section{Lise St-Georges}

Volume 39, numéro 3, hiver 1986

URI : https://id.erudit.org/iderudit/304370ar

DOI : https://doi.org/10.7202/304370ar

Aller au sommaire du numéro

Éditeur(s)

Institut d'histoire de l'Amérique française

\section{ISSN}

0035-2357 (imprimé)

1492-1383 (numérique)

Découvrir la revue

Citer cet article

St-Georges, L. (1986). Commerce, crédit et transactions foncières : pratiques de la communauté marchande du bourg de l'Assomption, 1748-1791. Revue d'histoire de l'Amérique française, 39(3), 323-343.

https://doi.org/10.7202/304370ar d'utilisation que vous pouvez consulter en ligne. 


\title{
COMMERCE, CRÉDIT ET TRANSACTIONS FONCIERES: PRATIQUES DE LA COMMUNAUTÉ MARCHANDE DU BOURG DE L'ASSOMPTION, 1748-1791
}

\author{
LISE ST-GEORGES \\ Département d' histoire \\ Université du Québec à Montréal
}

\section{INTRODUCTION}

L'étude des marchands ruraux, dans la seconde moitié du $18 \mathrm{e}$ siècle, permet de saisir un aspect important du développement du capitalisme commercial dans les campagnes québécoises. La plupart de ces marchands résident dans les villages dont plusieurs se forment d'ailleurs à cette époque. L'historiographie s'est surtout intéressée à l'analyse des activités commerciales, financières et foncières de certains de ces marchands ruraux ${ }^{2}$. Pour notre part, nous voulons appréhender l'action marchande par le biais d'un groupe, d'une collectivité plutôt qu'au moyen d'études individuelles. Cette approche repose, à l'instar des enquêtes individuelles, sur l'exploitation des archives notariales. Elle se distingue cependant par la méthodologie utilisée. Nous avons dépouillé systématiquement non seulement tous les actes concernant les marchands demeurant au bourg de l'Assomption entre 1748 et 1791, mais également ceux contractés par les autres villageois. De plus nous avons constitué des séries complètes d'obligations, de mutations foncières et d'inventaires après décès paysans, dans l'ensemble de la région pour certaines années. Cette méthode nous permet à la fois d'identifier les activités des commerçants et de les comparer, dans les domaines du crédit et de la propriété foncière, à celles des autres groupes sociaux. Cette approche nous permet ainsi de mieux circonscrire l'étendue et le poids de l'intervention marchande dans la région rurale.

Entre 1748 et 1791, une importante communauté marchande se forme et prospère au bourg de l'Assomption. Centre de pouvoirs reli-

\footnotetext{
1 Cet article est la version remaniée d'une partie d'un mémoire de maîtrise intitulé «Le village de l'Assomption, 1748-1791" (mémoire de maîtrise, Université du Québec à Montréal, département d'histoire, 1984). Il reprend le texte d'une communication présentée au congrès de la Société historique du Canada, à Montréal, en mai 1985. Je remercie mes co-directeurs, messieurs Richard Chabot et Jean-Claude Robert, de leurs conseils judicieux et de leur disponibilité.

2 A titre d'exemples, les articles de Louis Michel, «Un marchand rural en Nouvelle-France: François-Augustin Bailly de Messein 1709-1771», Revue d' histoire de l'Amérique française, 33,2 (septembre 1979): 215-62; «Le livre de compte (1784-1792) de Gaspard Massue, marchand à Varennes», Histoire sociale, 13,26 (nov. 1980): 369-98.
} 
gieux et civils, lieu d'échange de biens et de services, ce village constitue un mode d'insertion pour les marchands dans le monde agricole. Du bourg, ils exercent leurs activités économiques et leur influence sur un vaste espace rural regroupant les territoires des seigneuries de SaintSulpice, Lachenaie ${ }^{3}$, Lavaltrie et Repentigny. La dynamique de l'échange marchand, à l'instar de celle de l'ensemble de l'économie villageoise, demeure cependant tributaire du développement de l'économie agricole et de l'expansion démographique de cette zone rurale.

\section{A - LE VILLAGE DE L'ASSOMPTION}

\section{1 - Formation et développement}

Situé à quelque 40 kilomètres au nord-est de Montréal et occupant une presqu'île créée par un des méandres de la rivière l'Assomption, le village de l'Assomption se forme au coeur d'un réseau hydrographique composé des affluents de cette rivière. Ces cours d'eau constituent des axes privilégiés de pénétration de peuplement vers l'intérieur des terres ${ }^{4}$. Cette situation stratégique confère au bourg un rayonnement au-delà de la seigneurie de Saint-Sulpice ${ }^{5}$ dont il fait partie.

Malgré la construction d'une église en pierre dès 1749 et l'obtention, en 1754, d'une reconnaissance officielle ${ }^{6}$, le village de l'Assomption ne progresse que très lentement entre 1748 et 1769 . Cette période de latence résulte principalement du climat d'instabilité sociale et économique qui sévit tout au long de la guerre de Sept Ans et qui perdure au cours des premières années du régime anglais. Cette période est vécue dans l'incertitude par les Sulpiciens qui, seigneurs et ecclésiastiques, craignent la spoliation de leurs biens ${ }^{\text {. }}$

Le retour d'une relative stabilité, vers 1769 , se manifeste par la reprise des concessions dans les secteurs ruraux et villageois de la seigneurie de Saint-Sulpice. Au cours des décennies 1770 et 1780 , le village de l'Assomption et la zone rurale environnante se développent rapidement. Ainsi entre 1765 et 1791, la population du bourg augmente

\footnotetext{
Selon les époques, cette appelation regroupe également les fiefs Martel, Bailleul et la seigneurie de l'Assomption.

${ }_{4}$ R. Harris, The Seigneurial System in Early Canada (Québec, Presses de l'Université Laval, 1968), 127.

Cette seigneurie est concédée en 1640 au Séminaire de Saint-Sulpice de Paris par la Compagnie de la Nouvelle-France.

«Ordonnance qui établit un village à l'Assomption, de deux (sic) arpens et demi de front sur sept arpens de profondeur, sur une terre appartenant au curé; du dix-huitième mars, mil sept cens cinquante quatre», signée par Duquesne et Bigot, Édits, ordonnances royaux au Canada, tome 2 (Québec, R. Fréchette, 1855), 415.

O. Maurault, Nos Messieurs (Montréal, Éditions du Zodiaque, 1936), 28-29.
} 
de plus de $368 \%{ }^{8}$ comparativement à $228 \%$ pour la région immédiate ${ }^{9}$ et à un accroissement de $131 \%{ }^{10}$ pour l'ensemble de la colonie. La forte croissance obtenue par le village s'explique par le caractère embryonnaire qu'il possède encore en 1765 alors que la zone rurale accueille déjà des habitants depuis plusieurs décennies. Ces accroissements de la population participent au mouvement de colonisation et d'expansion démographique que connaît alors l'ensemble de la plaine de Montréal.

TABLEAU 1

Ventilation socio-professionnelle des résidants du bourg de l'Assomption en 1781 et en 1791

\begin{tabular}{|c|c|c|}
\hline \multirow{2}{*}{ Catégories } & \multicolumn{2}{|c|}{ Nombre de chefs de ménage } \\
\hline & 1781 & 1791 \\
\hline marchands & 12 & 15 \\
\hline artisans : bois et construction & 11 & 17 \\
\hline métaux & 7 & 5 \\
\hline cuir & 5 & 6 \\
\hline vêtement & 3 & 4 \\
\hline alimentation & 1 & 1 \\
\hline divers & 2 & 2 \\
\hline professions libérales & 5 & 6 \\
\hline habitants & 4 & 2 \\
\hline rentiers & 5 & 7 \\
\hline autres & 3 & 6 \\
\hline indéfinis & 7 & 11 \\
\hline TOTAUX & 65 & 82 \\
\hline
\end{tabular}

Source: Fichier-ménage pour le village de l'Assomption.

8 A savoir 10 ménages en 1765,73 en 1784 et 82 en 1791 . Nombres établis à partir de notre fichier-ménage qui regroupe l'ensemble des renseignements concernant chaque résidant et/ ou propriétaire du bourg de l'Assomption entre 1748 et 1791 . Nous avons établi des fiches à partir du dépouillement systématique des archives seigneuriales et des greffes des notaires ayant pratiqué dans la région. Nous y avons également inscrit les informations recueillies dans l'histoire locale et les registres paroissiaux.

9 La région regroupe les paroisses de Saint-Pierre-du-Portage, de la Purification-de-laBienheureuse-Vierge-Marie de Repentigny, de Saint-Antoine-de-Lavaltrie, de Saint-Paul-deLavaltrie, de Saint-Jacques-de-l'Achigan et de Saint-Roch-de-l'Achigan. La population de cette région se chiffre en 1765 à 2391 individus, en 1784 à 5168 personnes et à 7853 en 1791 . «Recensements de 1765 et 1791», Recensements du Canada 1665-1871, vol. IV; «Recensement de 1784», cité dans F. Ouellet, «Libéré ou exploité: le paysan québécois d'avant 1850», Histoire sociale, 13 (1980): 339-368.

${ }_{10}$ F. Ouellet, Le Bas-Canada 1791-1840. Changements structuraux et crise (Ottawa, Presses de l'Université d'Ottawa, 1980), 23. 


\section{2 - Les villageois : ventilation socio-professionnelle}

L'essor démographique et économique de la région de l'Assomption s'accompagne d'une augmentation de la demande pour les biens et les services. Au village résident principalement des artisans et des marchands (tableau 1). Ainsi retrouvons-nous plusieurs artisans oeuvrant dans les secteurs du bois, de la construction et à l'accomplissement de divers travaux publics, dont la construction de l'église en pierre au bourg de l'Assomption. L'augmentation de la population accroît également les demandes pour les produits de consommation domestique tels les chaussures, les vêtements et autres.

La communauté marchande profite aussi de cet accroissement du marché rural qui lui procure des revenus substantiels. Elle constitue en fait le groupe social le plus fortuné à l'Assomption, dans la seconde moitié du $18 \mathrm{e}$ siècle. L'augmentation démographique offre également une clientèle potentielle aux membres des professions libérales (notaires, médecins) dont le nombre apparaît relativement important. D'autre part, le bourg n'accueille que peu de cultivateurs, qu'ils soient retraités ou actifs, et de rentiers. Au 18e siècle, ce village se caractérise donc particulièrement par ses fonctions d'échange.

\section{B - LA COMMUNAUTÉ MARCHANDE DE L'ASSOMPTION 1 - L'effectif et l'ethnicité}

Entre 1748 et 1791 , la communauté marchande regroupe plus de 15 pour cent des chefs de ménage, soit 25 des 165 qui résident au bourg de l'Assomption. Leur nombre croît principalement à partir des années 1770. Au moment de la Conquête, cinq villageois se déclarent commerçants; en 1771, l'effectif se maintient à six alors qu'en 1781, il grimpe à 12 et en 1791 , à 15 . Les périodes d'activité de ces commerçants varient énormément. Certains, souvent parmi les plus prospères, y exercent pendant toute leur carrière. A ce noyau s'ajoutent des marchands qui, commerçant déjà dans la région montréalaise et attirés par la croissance du village, viennent s'y installer définitivement dans les années 1770 . D'autre part, certains jeunes commerçants, souvent apparentés à un marchand de l'Assomption, ont de la difficulté à s'imposer et quittent rapidement le bourg pour tenter leur chance dans le secteur rural environnant ou dans une autre région.

Les membres de la communauté marchande se distinguent entre eux par diverses caractéristiques dont l'ethnicité, la nature de leurs activités économiques, le niveau de fortune et le prestige social. A la douzaine de Canadiens francophones ${ }^{11}$ s'ajoutent cinq Français de naissance, six commerçants d'origine britannique et deux individus dont nous ignorons l'ethnicité. Alors que les Français s'installent à l'As-

\footnotetext{
11 Trois marchands sont nés au bourg, six proviennent de l'île de Montréal, deux de la rive sud de Montréal et un de la ville de Québec.
} 
somption au cours de la dernière décennie du régime français, les marchands britanniques s'y établissent surtout durant les années 1780. Leur venue est principalement motivée par le commerce des fourrures. D'ailleurs, certains d'entre eux sont liés à la compagnie du Nord-Ouest dont George McBeath ${ }^{12}$ et David McCrae ${ }^{13}$. A ces derniers, s'ajoutent toutefois certains francophones, entre autres: André Blondeau-Drézy ${ }^{14}$ et à la fin du $18 \mathrm{e}$ siècle, des enfants des marchands villageois de la première génération: François-Antoine Larocque fils et Laurent Leroux ${ }^{15}$.

\section{2 - Les activités commerciales et les niveaux de fortune}

Entre 1748 et 1791, l'activité principale de la majorité des marchands de l'Assomption demeure néanmoins le commerce de détail de produits locaux ou importés. Ce type d'échange donne lieu à une concurrence vive entre les marchands villageois dans l'espace rural environnant. L'étude des obligations, contractées envers ces derniers par les résidants de la région de 1782 à 1791 , livre une image partielle de cette concurrence (voir Tableau 2). Ce reflet demeure limité car seulement une partie des comptes courants sont transformés en obligations. Cette analyse permet cependant de circonscrire la «spécialisation» territoriale qui s'établit entre certains marchands prospères du bourg.

Ainsi, nous constatons le poids exercé par Germain Leroux, sur les créances du bourg et de la zone rurale de la seigneurie de SaintSulpice. Installé au village depuis la fin des années 1750, il oppose une forte compétition aux nouveaux détaillants qui tentent de s'établir dans les années 1770 et 1780 . Ces derniers s'implantent d'ailleurs avec plus d'aisance dans les seigneuries de Lachenaie, Lavaltrie et Repentigny, secteurs plus éloignés du village où ils subissent cependant la concurrence des marchands des côtes.

L'existence de la concurrence entre les commerçants de détail et l'étendue du territoire concerné, soit un rayon d'une vingtaine de kilomètres, nous incitent à nuancer l'image du marchand général sédentaire approvisionnant de son magasin, voisin de l'église, une clientèle rurale captive. Afin de rejoindre plus de paysans, le détaillant villageois doit sans doute trouver une façon de solliciter la clientèle soit directement à domicile, soit en déléguant un commis ou encore en établissant des comptoirs dans la zone rurale. Cependant à l'Assomption au 18e siècle, aucune source ne révèle l'existence de tels comptoirs.

\footnotetext{
12 F. Ouellet, «George McBeath», dans Dictionnaire biographique du Canada, vol. 5 (Québec, Presses de l'Université Laval, 1983), 563.

${ }_{13}$ ANQM, minute J. Beek, assignment, 11 octobre 1787 , no 320.

14 ANQM, minute J. Raymond, engagement, 20 février 1786 , no 321.

15 Ces derniers seront également membres co-fondateurs de la Banque de Montréal en 1819. A Lamalice, «François-Antoine Larocque» dans Dictionnaire biographique du Canada, vol. 9 (Québec, Presses de l'Université Laval, 1977), 501-502.
} 
TABLEAU 2

Répartition géographique des obligations contractées envers des marchands de l'Assomption,

par lieu de résidence du débiteur, 1782-1791

(en nombre d'obligations et en pourcentage)

\begin{tabular}{|c|c|c|c|c|c|c|c|c|}
\hline & $\begin{array}{c}\text { Bourg } \\
\text { du } \\
\text { Portage }\end{array}$ & $\begin{array}{l}\text { Seigneurie } \\
\text { de } \\
\text { St-Sulpice }\end{array}$ & $\begin{array}{c}\text { Seigneurie } \\
\text { de } \\
\text { Lachenaie }\end{array}$ & $\begin{array}{c}\text { Seigneurie } \\
\text { de } \\
\text { Lavaltrie }\end{array}$ & $\begin{array}{l}\text { Seigneurie } \\
\text { de } \\
\text { Repentigny }\end{array}$ & $\begin{array}{c}\text { Autres } \\
\text { seigneuries }\end{array}$ & $\begin{array}{l}\text { Lieux } \\
\text { de } \\
\text { résidence } \\
\text { indéfinis }\end{array}$ & Total \\
\hline LEROUX, Germain & $\begin{array}{c}18 \\
(47,4)^{*}\end{array}$ & $\begin{array}{c}65 \\
(63,1)\end{array}$ & $\begin{array}{c}26 \\
(32,5)\end{array}$ & $\begin{array}{c}8 \\
(19,5)\end{array}$ & $\begin{array}{c}2 \\
(33,3)\end{array}$ & $\begin{array}{c}2 \\
(28,57)\end{array}$ & $\begin{array}{c}2 \\
(33,3)\end{array}$ & $\begin{array}{c}123 \\
(43,8)\end{array}$ \\
\hline $\begin{array}{l}\text { LAROCQUE, François- } \\
\text { Antoine }\end{array}$ & $\begin{array}{c}11 \\
(28,9)\end{array}$ & $\begin{array}{c}24 \\
(23,3)\end{array}$ & $\begin{array}{c}38 \\
(45,0)\end{array}$ & $\begin{array}{c}17 \\
(41,4)\end{array}$ & $\begin{array}{c}4 \\
(66,7)\end{array}$ & $\begin{array}{c}2 \\
(28,57)\end{array}$ & $\begin{array}{c}1 \\
(16,7)\end{array}$ & $\begin{array}{c}97 \\
(34,5)\end{array}$ \\
\hline VIGER, Joseph & $\begin{array}{c}5 \\
(13,2)\end{array}$ & $\begin{array}{c}4 \\
(3,9)\end{array}$ & $\begin{array}{c}2 \\
(2,5)\end{array}$ & $\begin{array}{c}12 \\
(29,3)\end{array}$ & - & $\begin{array}{c}1 \\
(14,33)\end{array}$ & $\begin{array}{c}1 \\
(16,7)\end{array}$ & $\begin{array}{c}25 \\
(8,9)\end{array}$ \\
\hline $\begin{array}{l}\text { BRUGUIÈRE, Jean- } \\
\text { Baptiste }\end{array}$ & $\begin{array}{c}3 \\
(7,9)\end{array}$ & $\begin{array}{c}6 \\
(5,8)\end{array}$ & $\begin{array}{c}7 \\
(8,8)\end{array}$ & $\begin{array}{c}2 \\
(4,9)\end{array}$ & - & $\begin{array}{c}1 \\
(14,33)\end{array}$ & - & $\begin{array}{c}19 \\
(6,8)\end{array}$ \\
\hline CORRY, Thomas & $\begin{array}{c}1 \\
(2,6)\end{array}$ & $\begin{array}{c}3 \\
(2,9)\end{array}$ & $\begin{array}{c}5 \\
(6,2)\end{array}$ & $\begin{array}{c}2 \\
(4,9)\end{array}$ & - & $\begin{array}{c}1 \\
(14,33)\end{array}$ & $\begin{array}{c}2 \\
(33,3)\end{array}$ & $\begin{array}{c}14 \\
(5,0)\end{array}$ \\
\hline Autres marchands & - & $\begin{array}{c}1 \\
(1,0)\end{array}$ & $\begin{array}{c}2 \\
(2,5)\end{array}$ & - & - & - & - & $\begin{array}{c}3 \\
(1,0)\end{array}$ \\
\hline TOTAL & $\begin{array}{c}38 \\
(100)\end{array}$ & $\begin{array}{c}103 \\
(100)\end{array}$ & $\begin{array}{c}80 \\
(100)\end{array}$ & $\begin{array}{c}41 \\
(100)\end{array}$ & $\begin{array}{c}6 \\
(100)\end{array}$ & $\begin{array}{r}7 \\
(100)\end{array}$ & $\begin{array}{c}6 \\
(100)\end{array}$ & $\begin{array}{c}281 \\
(100)\end{array}$ \\
\hline
\end{tabular}

* Pourcentage d'obligations signées envers ce marchand dans ce secteur géographique.

Source: 281 obligations signées par des résidents du bourg et de la région de l'Assomption. 
Au commerce de détail, s'ajoutent également ceux du bois, des céréales et des fourrures. De ces activités commerciales, découlent parfois des interventions dans le domaine du crédit et de la propriété foncière rurale. Certains individus s'engagent dans plusieurs secteurs d'activités alors que d'autres se restreignent à un ou deux champs d'intervention économique. Tous ne profitent pas également de ces différentes activités. Ainsi les inventaires après décès témoignent des écarts de fortune existant parmi la communauté marchande.

Durant la période observée, dix de nos marchands villageois sont soumis à un inventaire après décès de leurs biens. Les renseignements relatifs aux âges recueillis dans les contrats de mariage et dans les registres d'état civil révèlent, qu'au moment de la rédaction de l'inventaire après décès, la plupart d'entre eux se situent en pleine période d'activité soit entre 40 et 50 ans.

Les fortunes possédées par les marchands de l'Assomption excèdent de beaucoup celles des autres villageois. La moyenne des actifs mobiliers et immobiliers détenus par les commerçants est d'environ 45000 livres $^{16}$, soit cinq fois supérieurs à la moyenne des actifs possédés par les membres des professions libérales et plus de dix fois supérieurs à ceux appartenant aux autres résidants du bourg ${ }^{17}$. Les marchands se distinguent à tous les niveaux: biens de consommation, instruments de production, créances et immeubles. Leur statut privilégié se reflète également dans leurs résidences qui sont souvent plus vastes et mieux aménagées que celles des autres villageois.

Néanmoins, il existe des disparités entre les marchands au niveau de la composition et de la valeur des actifs mobiliers (Tableau 3). Cette valeur connaît des écarts de l'ordre de 1 à 50. Ainsi, Pierre-Médard Pétrimoulx (M-4) représente le cas extrême d'un commerçant, possédant peu de marchandises et aucune créance, et agissant probablement à titre d'intermédiaire ou de commis pour un autre marchand local ou forain. Par contre, les François-Antoine Larocque (M-8), Thomas Corry (M-9) et Germain Leroux (M-10) ont des fortunes reflétant la prospérité de leurs activités commerciales et financières : marchandises et créances importantes et dans le cas de Leroux, la possession d'un numéraire, soit 12152 livres, dépassant celui détenu par l'ensemble des autres marchands inventoriés. Cependant, malgré les écarts de fortune séparant ses membres, la communauté marchande demeure le groupe social villageois le plus fortuné. Le moins prospère des marchands inventoriés, Pierre-Médard Pétrimoulx (M-4), jouit néanmoins d'une fortune

\footnotetext{
16 Toutes les valeurs monétaires exprimées dans cet article le sont en livres ancien cours. 7 Soit une moyenne de l'actif des biens meubles et immeubles des membres de professions libérales de 9114 livres; des artisans de 1000 livres; des habitants de 888 livres; et des indéfinis de 419 livres. L'unique rentier pour lequel nous avons un inventaire possède un actif évalué à 1 882 livres.
} 
TABLEAU 3

Composition de la fortune de dix marchands ayant résidé à l'Assomption entre 1748 et 1791

\begin{tabular}{|c|c|c|c|c|c|c|c|c|}
\hline & $\begin{array}{c}\text { Biens de } \\
\text { consommation }\end{array}$ & $\begin{array}{l}\text { Instruments de } \\
\text { travail, cheptel } \\
\text { et marchandises }\end{array}$ & Numéraire & Créances & Immeubles & Actif & $\begin{array}{c}\text { Dettes } \\
\text { passives }\end{array}$ & Bilan \\
\hline Marchand-1 & 778 & 9532 & - & 31931 & 14000 & 56241 & $?$ & $?$ \\
\hline Marchand-2 & 1469 & 8896 & - & 3000 & 12275 & 25640 & 3586 & 22054 \\
\hline Marchand-3 & 2590 & 5707 & 3600 & - & 18200 & 30097 & 7536 & 22561 \\
\hline Marchand-4 & 369 & 306 & 73 & - & 1041 & 1789 & 814 & 875 \\
\hline Marchand-5 & 3430 & 6519 & - & 8916 & 9600 & 28465 & $?$ & $?$ \\
\hline Marchand-6 & 2588 & 1312 & 268 & 4028 & 2100 & 10296 & 9185 & 1111 \\
\hline Marchand-7 & 2677 & 4146 & 162 & 9748 & 1300 & 18033 & 26483 & -8450 \\
\hline Marchand-8 & 2117 & 33837 & 1334 & 38595 & 26466 & 102340 & 76374 & 25974 \\
\hline Marchand-9 & 4555 & 12361 & 469 & 59940 & 17508 & 94833 & 51707 & 43126 \\
\hline Marchand-10 & 4637 & 10283 & 12152 & 37424 & 18500 & 82996 & - & 82996 \\
\hline Moyennes & 2521 & 9290 & 1806 & 19358 & 12099 & 45074 & $\begin{array}{c}21961 \\
(8 / 10)\end{array}$ & $\begin{array}{c}23781 \\
(8 / 10)\end{array}$ \\
\hline
\end{tabular}

Source: 10 inventaires après décès de marchands, 1767-1792. 
supérieure à celle de la majorité des villageois qui ne sont ni marchands, ni professionnels.

\section{3 - Le prestige social}

$\mathrm{Au}$ pouvoir économique des marchands correspond un prestige social qui se concrétise par le cumul de diverses charges civiles. Ne subissant pas la compétition du seigneur qui, dans la seigneurie de Saint-Sulpice, est une communauté religieuse, les commerçants occupent différentes tâches civiles et juridiques qui contribuent à consolider leur influence. Ainsi, François-Antoine Larocque, d'abord juge de paix, est élu député du comté de Leinster en 1791. Après son décès l'année suivante (31 octobre 1792) ${ }^{18}$, c'est un autre marchand, George McBeath qui le remplace en $1793^{19}$. Ce dernier est défait le 20 juillet 1796 par un autre marchand du bourg: Joseph Viger, membre du Bloc canadien ${ }^{20}$. Le fait que les trois premiers députés du comté de Leinster soient des commerçants de l'Assomption témoigne de l'importance de ce bourg et de sa communauté marchande dans la région environnante.

Pour sa part, Germain Leroux occupe les postes de juge de paix et de commissaire de la Cour de requête, fonction qu'il partage, en 1791, avec les commerçants George McBeath et Toussaint Pothier ${ }^{21}$. Les marchands demeurent cependant absents des fonctions paroissiales. Les postes de syndic ou de marguillier sont plutôt comblés par certains artisans villageois ou par des paysans aisés ${ }^{22}$. Néanmoins, au village de l'Assomption, durant toute la seconde moitié du $18 \mathrm{e}$ siècle, la communauté marchande prédomine socialement grâce à sa puissance économique.

\section{C - RAPPORTS ÉCONOMIQUES ENTRE LES MARCHANDS ET LES PAYSANS : CRÉDIT ET TRANSACTIONS FONCIERES}

\section{1 - Structure de l'endettement paysan 1765-1769, 1775-1779 et 1785-1789}

L'importance du prélèvement marchand dans l'endettement paysan transparaît de l'étude des dettes passives inscrites dans les inventaires après décès paysans de la région de l'Assomption pour les années 1765-1769, 1775-1779 et 1785-1789 (voir tableau 4). Tout au cours de ces trois périodes quinquennales, les créances réclamées par les marchands des côtes et du bourg représentent au moins le tiers des dettes

\footnotetext{
18 M. Fournier, La représentation parlementaire de la région de Joliette (Joliette, Société historique de Joliette, 1977), 164.

19 Ibidem, 181 .

20 Ibidem, 219

21 ANQM, minute B. Faribault, demande d'un règlement de police pour le bourg de l'Assomption, ler juillet 1791 .

22 Roy, Histoire de l'Assomption, 80.
} 
TABLEAU 4

Ventilation, par catégorie de créanciers, des dettes passives recensées dans cent-sept inventaires après décès de ménages paysans de la région de l'Assomption 1765-1769, 1775-1779 et 1785-1789 (en livres)

\begin{tabular}{|c|c|c|c|c|c|c|c|c|c|c|c|c|c|}
\hline$\frac{\mathscr{U}}{0}$ & है & 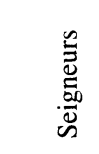 & 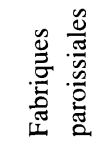 & 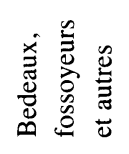 & 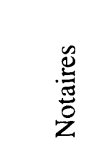 & 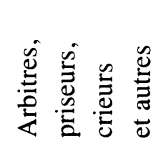 & $\frac{\mathscr{n}}{\tilde{J}}$ & 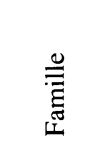 & 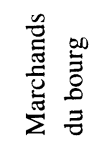 & 旁总 & 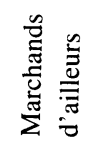 & 竞 & 要 \\
\hline $\begin{array}{c}1765-1769 \\
\%\end{array}$ & 22 & $\begin{array}{r}800 \\
(8,71)\end{array}$ & $\begin{array}{r}407 \\
(4,43)\end{array}$ & $\begin{array}{c}27 \\
(0,29)\end{array}$ & $\begin{array}{c}700 \\
(7,62)\end{array}$ & $\begin{array}{c}45 \\
(0,49)\end{array}$ & $\begin{array}{c}173 \\
(1,88)\end{array}$ & $\begin{array}{c}1122 \\
(12,21)\end{array}$ & $\begin{array}{r}2381 \\
(25,91)\end{array}$ & $\begin{array}{c}202 \\
(2,20)\end{array}$ & $\begin{array}{r}937 \\
(10,2)\end{array}$ & $\begin{array}{c}2395 \\
(26,06)\end{array}$ & $\begin{array}{r}9189 \\
(100 \%)\end{array}$ \\
\hline $\begin{array}{c}1775-1779 \\
\%\end{array}$ & 43 & $\begin{array}{r}798 \\
(3,81)\end{array}$ & $\begin{array}{r}641 \\
(3,06)\end{array}$ & $\begin{array}{c}55 \\
(0,26)\end{array}$ & $\begin{array}{c}2586 \\
(12,33)\end{array}$ & $\begin{array}{c}247 \\
(1,18)\end{array}$ & $\begin{array}{c}613 \\
(2,92)\end{array}$ & $\begin{array}{c}2037 \\
(9,71)\end{array}$ & $\begin{array}{r}3800 \\
(18,13)\end{array}$ & $\begin{array}{c}741 \\
(3,53)\end{array}$ & $\begin{array}{c}3308 \\
(15,77)\end{array}$ & $\begin{array}{r}6144 \\
(29,3)\end{array}$ & $\begin{array}{l}20970 \\
(100 \%)\end{array}$ \\
\hline $\begin{array}{c}1785-1789 \\
\%\end{array}$ & 42 & $\begin{array}{l}2167 \\
(6,94)\end{array}$ & $\begin{array}{l}1149 \\
(3,68)\end{array}$ & $\begin{array}{c}24 \\
(0,08)\end{array}$ & $\begin{array}{c}4241 \\
(13,59)\end{array}$ & $\begin{array}{c}322 \\
(1,03)\end{array}$ & $\begin{array}{c}871 \\
(2,79)\end{array}$ & $\begin{array}{c}2525 \\
(8,09)\end{array}$ & $\begin{array}{c}10660 \\
(34,16)\end{array}$ & $\begin{array}{c}367 \\
(1,18)\end{array}$ & $\begin{array}{c}3462 \\
(11,1)\end{array}$ & $\begin{array}{c}5417 \\
(17,36)\end{array}$ & $\begin{array}{l}31205 \\
(100 \%)\end{array}$ \\
\hline
\end{tabular}


passives. La part des marchands villageois varie entre 18 et 34 pour cent alors que celle des autres commerçants oscille entre 10 et 16 pour cent.

Alors que le volume de l'endettement paysan fluctue selon les conjonctures économiques, sa structure varie peu. Tout au plus remarquons-nous que les arrérages seigneuriaux sont mieux acquittés en période de stabilité économique, telle celle de 1775-1779. A l'inverse, une période de mauvaises récoltes, telles les années 1785-1789, voit la part relative des marchands augmenter dans les dettes paysannes, résultant de l'incapacité croissante de plusieurs familles paysannes à acquitter rapidement leurs dettes de consommation. Cette hausse de la part marchande s'accompagne de la diminution de celle de la catégorie «autres créanciers». La diminution de la production comme celle du revenu agricole n'encouragent nullement l'engagement de maind'oeuvre supplémentaire ou l'achat de terres, composantes importantes des dettes recensées dans cette catégorie.

\section{2 - Obligations consenties à des marchands du bourg 1753-1791}

Du commerce de détail exercé par des marchands de l'Assomption découlent souvent des comptes courants impayés dont les créanciers exigent la transformation en obligations. Le paiement de la dette est alors garanti par la présence d'une hypothèque générale et/ou spéciale. Elle s'accompagne également, dans la majorité des cas, de l'octroi de l'intérêt légal de $6 \%$. La presque totalité des obligations signées par les habitants de la région envers les marchands du bourg le sont d'ailleurs pour marchandises vendues.

La figure 1 représente les nombres annuels d'obligations signées à des marchands de l'Assomption entre 1753 et 1791 . Nous remarquons que les mouvements de cette courbe sont interprétables à partir des différentes conjonctures régionales ou coloniales. Ainsi la faiblesse du marché des obligations avant la fin des années 1760 manifeste le caractère embryonnaire du village et l'instabilité du climat politique et économique de la colonie.

Si l'on accepte que «le mouvement annuel des obligations a une signification économique ${ }^{23}$ et qu'en période de crise, l'augmentation de leur nombre résulte d'un accroissement de l'endettement rural, nous pouvons présumer que 1767, 1768 et 1769 sont des années de conjoncture défavorable dans la région de l'Assomption. La hausse du prix du blé confirme cette hypothèse. Dans les paroisses de Berthier, de Lache-

\footnotetext{
23 Serge Dontenwill, «Rapports ville-campagne et espace économique microrégional. Cherlieu et son plat-pays au XVIIIe siècle», dans Centre d'histoire économique et sociale de la région lyonnaise, Villes et campagnes XVe-XXe siècles (Lyon, Presses universitaires de Lyon, 1977), 151 .
} 
naie, de Repentigny, de l'Ile Dupas et de l'Assomption, le minot de blé atteint le seuil de 6 livres en 1769 alors qu'auparavant il se maintenait à environ 3 ou 4 livres ${ }^{24}$. Cette augmentation témoigne-t-elle réellement des conditions néfastes vécues sur la rive nord de Montréal durant ces années ou plutôt d'une hausse de la demande de blé conséquente aux mauvaises récoltes dans la région de Québec ${ }^{25}$ ?

D'autre part, la reprise du mouvement de colonisation dans les années 1770 favorise l'augmentation de la production agricole et stimule les échanges commerciaux sans toutefois alourdir l'endettement paysan. Cette période de bonne conjoncture, commune à l'ensemble de la colonie, profite également de l'ouverture des marchés extérieurs au blé canadien ${ }^{26}$.

La hausse du nombre des obligations au début de la décennie 1780 résulte d'une succession de mauvaises récoltes que connaît le Québec entre 1780 et $1784^{27}$. Les paysans doivent alors ajouter à leurs achats habituels des quantités additionnelles de denrées alimentaires et de grains de semence. Ne pouvant rembourser leurs achats en raison de la baisse de la production agricole, ils s'endettent envers les marchands ruraux qui, talonnés eux-mêmes par leurs fournisseurs, exigent de leur clientèle la signature d'obligations. Ainsi, à l'Assomption, 15 des 25 obligations consenties à Joseph Viger le sont en 1783, année où ce négociant doit établir une cession de ses biens en faveur de ses créanciers montréalais ${ }^{28}$.

La fin de la décennie 1780 connaît également de mauvaises récoltes et conséquemment, une hausse des prix céréaliers. De 1788 à 1789 , le prix du minot de blé dans la paroisse de l'Assomption augmente de 3 livres 16 sols à 10 livres $^{29}$, témoignant d'un malaise évident dans la production agricole. Tout comme en 1784, Germain Leroux attend, en 1789, au lendemain de la crise, pour exiger la signature d'obligations par ses clients. Il incite alors certains paysans dont les comptes sont

${ }^{24}$ F. Ouellet en collaboration avec J. Hamelin et R. Chabot, «Les prix agricoles dans les villes et les campagnes du Québec d'avant 1850: aperçus quantitatifs», Histoire sociale, 15,29 (mai 1982): 117 .

25 F. Ouellet, Histoire économique et sociale du Québec 1760-1850, tome 1 (Montréal, Fides, 1971), 83 ses de l'Université d'Ottawa, 1976), 11 .

27 Ouellet, Histoire économique..., 111.

28 En $1783, \mathrm{~J}$. Viger doit établir un acte de cession de ses biens ou acte d'atermoiement en faveur de ses créanciers envers lesquels il est redevable de plus de 30100 livres. Tout en demeurant propriétaire de ses biens, J. Viger doit soumettre la gestion de ces derniers à la tutelle d'un syndic nommé par ses créanciers. Il doit obtenir leur autorisation avant d'effectuer un nouvel investissement ou une vente de ses biens. D'autre part, jusqu'à ce que la dette soit résorbée, les créanciers peuvent exiger en tout temps la vente partielle ou totale des biens du débiteur.

29 Ouellet, «Les prix agricoles...», 117. 
fermés ou limités chez les autres marchands à s'approvisionner, en période de crise, à son magasin de l'Assomption ${ }^{30}$.

Indicateur utile pour identifier les fluctuations de l'économie régionale, les obligations doivent néanmoins être utilisées avec prudence. Entre autres, elles ne dévoilent que l'aspect officialisé de l'endettement dans une région. Parallèlement subsistent plusieurs formes de transactions économiques sous seing privé dont nous ne pouvons évaluer l'importance: compte courant, billet, promesse verbale et autres. Ainsi Thomas Corry, qui dispose pourtant d'un des plus forts actifs au sein de la communauté marchande ${ }^{31}$, ne se classe que cinquième au chapitre des obligations avec seulement $5,4 \%$ de la valeur totale des 408 obligations (voir tableau 5). Ce phénomène s'explique partiellement par le fait que le marchand transige à la fois dans le commerce de détail et dans celui des fourrures. Aussi présumons-nous que T. Corry ne recourt pas à l'obligation pour les dettes contractées par les clients qui sont également ses engagés à titre de voyageurs. Le marchand n'a alors qu'à prélever directement sur leurs gages le montant de leurs achats à son magasin.

D'autre part, la comparaison entre marchands, à partir des obligations, doit tenir compte de la période d'activité de l'individu. Le poids relatif de Jean-Baptiste Durocher est amoindri par le fait que son action marchande intervient au tout début de la formation du village et qu'elle est en bonne partie perturbée par la guerre de Sept Ans. Si nous le comparons aux autres marchands de son époque, nous constatons qu'il concentre à lui seul 49 des 91 obligations signées entre 1753 et 1777.

\section{3 - Prélèvement marchand dans l'endettement rural en 1784 et en 1787}

Afin de mieux observer les fluctuations du crédit marchand villageois, selon les conjonctures, par rapport à l'ensemble du crédit rural, nous avons dépouillé pour 1784 et 1787 toutes les obligations contractées par les résidants de la zone d'influence du bourg de l'Assomption. Nous pouvons ainsi comparer le poids de l'intervention marchande villageoise à celle des autres groupes de créanciers ruraux. L'année 1784 est caractérisée par de mauvaises récoltes, une épidémie et un prix relativement élevé du ble ${ }^{32}$. De plus, elle est, à l'Assomption, une année-

\footnotetext{
30 Cette opération est rendue possible par le fait que ce marchand de l'Assomption n'est nullement talonné par ses fournisseurs. Au contraire, il est le seul marchand à posséder, au moment de son décès en 1792, un compte excédentaire avec John McKindlay, négociant montréalais et principal fournisseur des marchands de l'Assomption.

31 Il possède alors un actif mobilier et immobilier évalué à 94833 livres auquel se greffe un passif de 51707 livres. ANQM, minute B. Faribault, inventaire après décès, ler octobre 1792 au 21 février 1793 .

32 Ouellet, Histoire économique..., 129.
} 
TABLEAU 5

Obligations consenties aux marchands du village de l'Assomption, 1753-1791, en nombre et en valeur (en livres françaises)

\begin{tabular}{|lccc|}
\hline Noms & $\begin{array}{c}\text { Nombre } \\
\text { d'obligations }\end{array}$ & Valeur & $\begin{array}{c}\text { \% valeur } \\
\text { totale }\end{array}$ \\
\hline Larocque, F.-A. (1780-1791) & 114 & 63114 & 34,8 \\
Leroux, G. (1762-1791) & 156 & 59552 & 32,8 \\
Durocher, J.-B. (1753-1777) & 49 & 15211 & 8,4 \\
Viger, J. (1782-1789) & 25 & 13358 & 7,4 \\
Corry, T. (1775-1791) & 17 & 9854 & 5,4 \\
Bruguière, J.-B. (1783-1791) & 19 & 5958 & 3,3 \\
Chastelain, A. (1766-1767) & 3 & 4859 & 2,7 \\
Thibaudeau, F. (1763-1769) & 5 & 3162 & 1,7 \\
Olivier, L. (1766-1776) & 6 & 2766 & 1,5 \\
Larocque, A. (1772-1776) & 8 & 2459 & 1,4 \\
Bruguière, C. (1782) & 2 & 621 & 0,3 \\
Baubin, C. (1785) & 1 & 347 & 0,2 \\
Richotte, P. (1753-1755) & $\underline{3}$ & 250 & 0,1 \\
TOTAUX & 408 & 181511 & 100 \\
\hline
\end{tabular}

Sources: 408 obligations, 1753-1791

record pour le nombre d'obligations signées envers des marchands du bourg. Si nous acceptons qu'en période de crise, l'endettement paysan croît, alors 1784 devrait être l'année la plus susceptible de nous présenter la part maximale des marchands dans le crédit rural. Par contre, une année de prospérité comme $1787^{33}$ devrait témoigner d'un marché financier plus calme où les paysans souffrent moins des pressions marchandes.

Les résultats obtenus nous obligent cependant à nuancer quelque peu ce raisonnement (voir tableau 6). En effet, seule la valeur absolue des créances marchandes villageoises croît en année de crise et non leur poids relatif. La part des commerçants de l'Assomption est toujours légèrement supérieure à $50 \%$ tant en 1784 qu'en 1787 . Les gros paysans, tout en quadruplant la valeur de leurs prêts notariés, profitent moins largement que les autres créanciers d'une période de crise. Ce $\frac{33}{17} 87$ est caractérisée par des conditions favorables et des prix céréaliers relativement
bas. Ouellet, Histoire économique..., 130. 
TABLEAU 6

Répartition des obligations signées par les résidants de la région de l’Assomption

en $\mathbf{1 7 8 4}$ et $\mathbf{1 7 8 7}$, par groupe de créanciers

(en livres françaises)

\begin{tabular}{|c|c|c|c|c|c|c|c|c|c|c|}
\hline \multirow[b]{2}{*}{ Année } & \multicolumn{2}{|c|}{$\begin{array}{l}\text { Marchands du } \\
\text { Bourg }\end{array}$} & \multicolumn{2}{|c|}{ Autres marchands } & \multicolumn{2}{|c|}{ Paysans } & \multicolumn{2}{|c|}{$\begin{array}{c}\text { Fabriques } \\
\text { paroissiales }\end{array}$} & \multicolumn{2}{|c|}{ Total } \\
\hline & Nombre & Valeur & Nombre & Valeur & Nombre & Valeur & Nombre & Valeur & Nombre & Valeur \\
\hline 1784 & $\begin{array}{c}55 \\
(56,12)\end{array}$ & $\begin{array}{l}24836 \\
(52,11)\end{array}$ & $\begin{array}{c}25 \\
(25,51)\end{array}$ & $\begin{array}{c}14331 \\
(30,07)\end{array}$ & $\begin{array}{c}17 \\
(17,35)\end{array}$ & $\begin{array}{c}7894 \\
(16,57)\end{array}$ & $\begin{array}{c}1 \\
(1,02)\end{array}$ & $\begin{array}{c}597 \\
(1,25)\end{array}$ & $\begin{array}{c}98 \\
(100 \%)\end{array}$ & $\begin{array}{l}47658 \\
(100 \%)\end{array}$ \\
\hline 1787 & $\begin{array}{c}10 \\
(55,56)\end{array}$ & $\begin{array}{r}4332 \\
(51,54)\end{array}$ & $\begin{array}{c}2 \\
(11,11)\end{array}$ & $\begin{array}{r}1773 \\
(21,09)\end{array}$ & $\begin{array}{c}5 \\
(27,78)\end{array}$ & $\begin{array}{c}1956 \\
(23,27)\end{array}$ & $\begin{array}{c}1 \\
(5,55)\end{array}$ & $\begin{array}{c}344 \\
(4,1)\end{array}$ & $\begin{array}{c}18 \\
(100 \%)\end{array}$ & $\begin{array}{r}8405 \\
(100 \%)\end{array}$ \\
\hline
\end{tabular}

Sources: 116 obligations signées par des résidants de la région de l'Assomption en 1784 et 1787. 
FIGURE 1

Nombres annuels d'obligations signées à des marchands de l'Assomption,

1753-1791 (408 obligations)

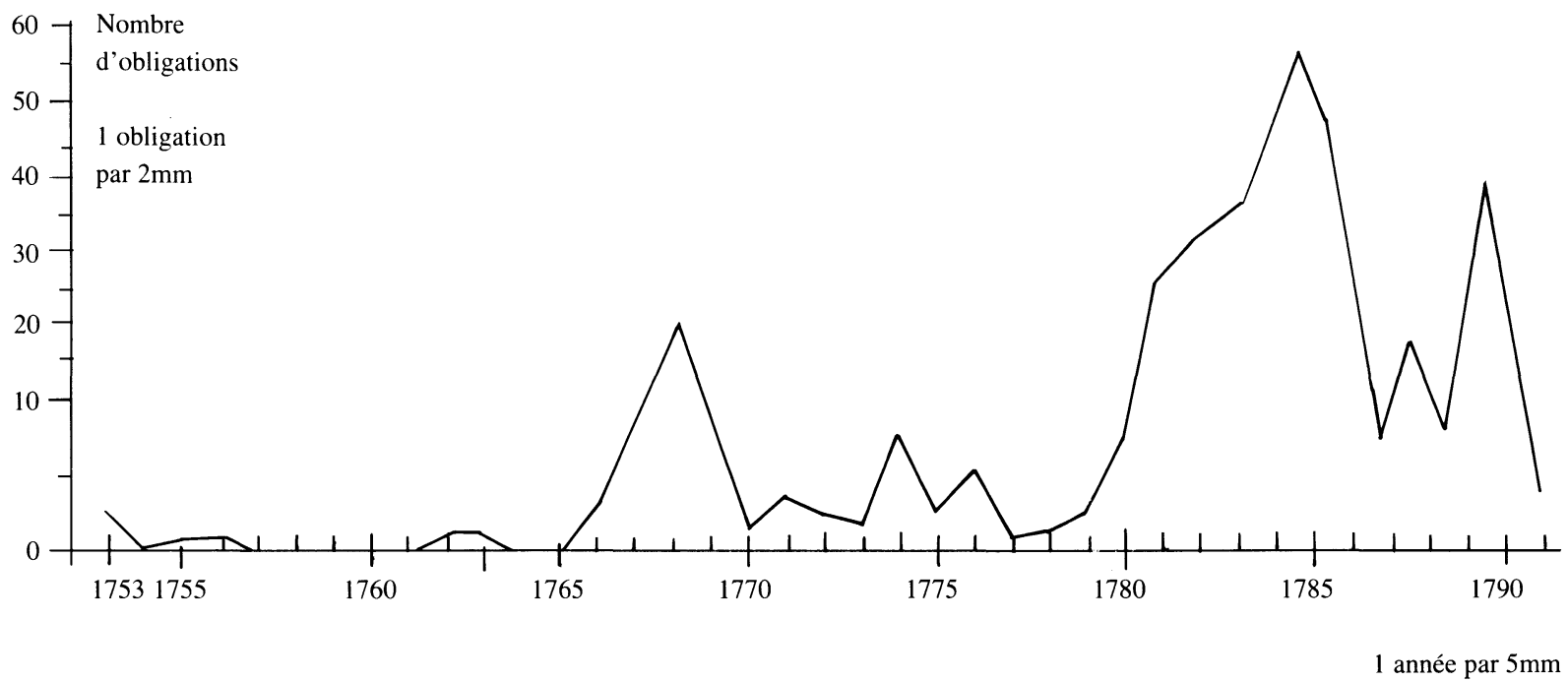


comportement reflète peut-être le choix des paysans prospères d'investir dans le marché foncier et de consolider leur patrimoine immobilier plutôt que d'intervenir dans le domaine du crédit, moins rentable à long terme ${ }^{34}$. Pour leur part, les marchands des côtes profitent de la rareté et de la cherté des grains pour augmenter leur emprise à l'endroit d'une clientèle rurale possédant de moins en moins de revenus et contrainte alors à s'endetter.

\section{4 - Formes de paiement, hypothèques et clauses spéciales}

La presque totalité des obligations signées envers les marchands de l'Assomption réclament un paiement en «espèces sonnantes». A l'occasion, le remboursement est exécuté sous une forme mixte : argent et céréales, numéraire et transport d'une dette due par un tiers. Le rachat de certaines obligations adopte la forme du travail non-rémunéré exécuté par le débiteur en faveur du créancier. En 1786, Joseph Pepin dit Moreau, cordonnier au bourg, signe une reconnaissance de dette pour un montant de 132 livres au marchand G. Leroux. Il s'engage auprès de ce dernier à le rembourser en travaillant pour lui «de son métier de cordonnier» à raison de 2 livres par jour. Le marchand fournit le matériel et accorde à l'artisan «en sus de la dite somme mentionnée le quart de son gain pour faire subsisté (sic) sa famille» ${ }^{35}$. Joseph Pepin se transforme ainsi en engagé de G. Leroux jusqu'à l'extinction de sa dette. Parfois même, les marchands se remboursent à la source en prélevant le montant de leurs créances sur les gages ou les salaires à venir des débiteurs.

Toutes les obligations sont garanties par une hypothèque générale s'appliquant à l'ensemble des actifs mobiliers et immobiliers des débiteurs. Elle est souvent accompagnée d'une hypothèque spéciale, identifiant, dans le cas des propriétaires ruraux et villageois, un bien foncier spécifique. Les non-propriétaires engagent, en caution, différents avoirs mobiliers: droits de succession, animaux, instruments de transport, et autres. Les artisans et les engagés hypothèquent parfois leur salaire ou leurs instruments de travail. Pour leur part, des métayers soumettent en caution leur part de fermage sur les récoltes et les animaux.

Des clauses spéciales s'ajoutent parfois aux hypothèques. Certaines stipulent le moment où s'effectuera une saisie ou une mise en location d'un bien hypothéqué à défaut du paiement. D'autres ressemblent à des hypothèques supplémentaires. Le débiteur s'engage alors à «conserver comme appartenant» au créancier des meubles, des instruments de travail ou des animaux. Le marchand F.-A. Larocque utilise beaucoup ce type de réserve sur les animaux lors de la crise de 1784 .

$34 \quad$ Voir infra, tableau 7, concernant le marché foncier en 1784 et en 1787.

35 ANQM, minute J. Raymond, obligation, 29 mai 1786, no 884. 
Ainsi, afin de garantir une obligation de 998 livres payable à ce négociant, Charles Chalue, habitant du ruisseau Saint-Pierre, hypothèque la terre de 90 arpents où il réside. Il s'engage également à «garder et à conserver exactement comme appartenant» à F.-A. Larocque: trois chevaux, deux vaches, deux boeufs, deux génisses, cinq cochons et six moutons. Le paysan ne peut ni vendre ni échanger ces animaux sans le consentement du créancier ${ }^{36}$. En période de crise prolongée, alors que les débiteurs sont incapables d'acquitter leurs obligations, le marchand pourra alors se prémunir de cette clause de réserve pour s'approprier officiellement le cheptel. Dans certains cas, ce dernier sera même confié à ferme chez son ancien propriétaire ${ }^{37}$.

Cependant, toutes ces modalités spéciales de paiement, d'hypothèque et de réserve demeurent marginales. La majorité des obligations répondent au modèle-type à savoir: paiement en numéraire et hypothèque spéciale adoptant la forme d'un bien foncier. Ces particularités nous révèlent cependant l'étendue des possibilités que détiennent les créanciers villageois pour récupérer leurs dûs sans recourir nécessairement à la vente par adjudication d'une propriété immobilière.

\section{5 - Activités foncières des marchands villageois dans le terroir rural de l'Assomption, 1784 et 1787}

L'historiographie établit fréquemment un lien entre le phénomène de l'endettement paysan et celui de l'appropriation du sol rural par des non-exploitants. Elle propose alors un mécanisme où des paysans, incapables de rembourser leurs dettes envers des créanciers villageois ou citadins, voient ces derniers acheter ou forcer la vente de la terre qu'ils avaient hypothéquée. L'exploitant rural perd ainsi son bien foncier qui est souvent son principal moyen de subsistance alors que les bourgeois augmentent leur emprise sur la zone rurale. Plusieurs études françaises ${ }^{38}$ se sont intéressées de cette manière à la question de l'emprise bourgeoise du sol pour la période pré-industrielle. Provoquant un drainage d'une part des revenus ruraux et une transformation de l'agriculture, l'action foncière des bourgeois témoigne alors de l'introduction du capitalisme dans le monde rural ${ }^{39}$. Certains auteurs, dont Robert Laurent et Pierre de Saint-Jacob, attribuent même à l'appropriation bour-

${ }^{36}$ ANQM, minute J. Raymond, obligation, 4 mai 1784 , no 465.

37 Dans la liste recensant le nom des paysans chez qui F.-A. Larocque a des animaux à ferme lors de son décès en 1792, nous retrouvons les noms de deux signataires d'obligations contenant cette clause de réserve sur les animaux. ANQTR, minute A.-I. Badeaux, 12 au 16 novembre 1792 , no 111 .

38 Entre autres celles de G. Cabourdin, Terres et hommes en Lorraine 1550-1635 (Nancy, Université de Nancy, 1977); S. Dontenwill, «Rapports ville-campagne...»; G. Roupnel, La ville et la campagne au XVIIIe siècle. Étude sur les populations du pays dijonnais (Paris, BellesLettres, 1960).

39 Pour une critique de cette idée, lire F. Braudel, Civilisation matérielle, économie et capitalisme XVe-XVIIIe siècles, tome 2 (Paris, Armand Colin, 1979), 287. 
geoise du terroir rural la prolétarisation des paysans, étape nécessaire à la révolution industrielle $e^{40}$. Cependant, nous pouvons nous interroger sur la pertinence d'accepter ce modèle comme étant explicatif de la réalité québécoise de la fin du $18 \mathrm{e}$ siècle.

Aussi, afin de vérifier si une filiation peut être établie entre l'endettement paysan et l'activité foncière villageoise dans la région de l'Assomption, nous avons opéré des dépouillements systématiques pour les années 1784 et 1787. Nous avons retenu tous les actes d'achat relatifs aux terres des seigneuries de Saint-Sulpice, de Lachenaie, de Lavaltrie et de Repentigny et qui se retrouvaient dans les greffes des notaires régionaux. Nous avons conservé les mêmes années-témoins que pour notre étude de l'endettement afin de mieux identifier les conséquences des périodes de crise et de prospérité sur le marché foncier. Ces actes de mutations foncières regroupent des ventes de gré à gré, représentant $79,1 \%$ des transactions en 1784 et $78 \%$ en 1787; des ventes de parts de succession dans une proportion de $19,5 \%$ en 1784 et de $22 \%$ en 1787 , et finalement une seule vente forcée faisant suite à un jugement obtenu en 1784 par le marchand F.-A. Larocque contre un de ses débiteurs ${ }^{41}$.

Le tableau 7 ne dévoile aucun signe d'appropriation marchande villageoise d'une part importante du terroir rural de la région de l'Assomption en 1784 et/ou 1787. La propriété paysanne prédomine largement tant en période de crise qu'en période de relative prospérité. $\mathrm{La}$ mauvaise conjoncture de 1784 s'accompagne, au niveau foncier, d'une augmentation de la quantité et de la valeur des terres vendues ${ }^{42}$. Les marchands, principaux créanciers de la région, ne profitent cependant pas du gonflement du marché foncier en 1784 pour augmenter leur part relative. Seuls les paysans capables non seulement de conserver leur patrimoine immobilier mais également de l'agrandir intensifient leur action foncière.

\section{6 - Les marchands villageois et la propriété rurale}

Les marchands de l'Assomption apparaissent témoigner peu d'intérêt pour l'acquisition de terres rurales en vue de la constitution d'un important patrimoine foncier. Souvent, ils se départissent assez rapidement des terres qu'ils acquièrent. Ils ne conservent alors qu'une ou deux exploitations, le plus souvent situées à proximité du bourg et baillées à ferme.

Entre 1748 et 1791 , peu de motivation existe, dans la région de l'Assomption, pour justifier des investissements marchands importants

\footnotetext{
$40 \quad \mathrm{R}$. Laurent, Les vignerons de la Côte d'Or au XIXe (Paris, Belles-Lettres, 1957), 45; P. de Saint-Jacob, Les paysans de la Bourgogne du nord au dernier siècle de l'Ancien Régime (Paris, Belles-Lettres, 1960), 569

41 ANQM, minute F. W. Gray, vente par adjudication, 30 septembre 1784, no 143.

42 La valeur moyenne d'un arpent transigé voisine les 22 livres en 1784 alors qu'elle n'est que de 14 livres en 1787 .
} 


\section{TABLEAU 7}

Répartition des achats de terres rurales transigées en 1784 et 1787 dans la région de l'Assomption par catégorie d'acheteur (superficie en arpents, valeur en livres françaises)

\begin{tabular}{|lcccccc|}
\hline & \multicolumn{2}{c}{$\begin{array}{c}\text { Nombre d'actes } \\
\text { \% }\end{array}$} & \multicolumn{2}{c}{$\begin{array}{c}\text { Superficie } \\
\text { \% }\end{array}$} & \multicolumn{2}{c|}{$\begin{array}{c}\text { Valeur } \\
\text { \% }\end{array}$} \\
\hline & $\mathbf{1 7 8 4}$ & $\mathbf{1 7 8 7}$ & $\mathbf{1 7 8 4}$ & $\mathbf{1 7 8 7}$ & $\mathbf{1 7 8 4}$ & $\mathbf{1 7 8 7}$ \\
\hline Paysans & 63 & 26 & 3386,5 & 1373,5 & 75325 & 16441 \\
& $(87,5)$ & $(76,48)$ & $(86,4)$ & $(73,9)$ & $(88,12)$ & $(62,08)$ \\
Marchands & 5 & 3 & 265 & 165 & 6105 & 1200 \\
villageois & $(6,94)$ & $(8,82)$ & $(6,76)$ & $(8,88)$ & $(7,14)$ & $(4,53)$ \\
Autres & 4 & - & 268 & - & 4050 & - \\
villageois & $(5,56)$ & - & $(6,84)$ & - & $(4,74)$ & - \\
Autres & - & 3 & - & 167,5 & - & 6600 \\
marchands* & - & $(8,82)$ & - & $(9,01)$ & - & $(24,9)$ \\
Artisans & - & 2 & - & 152,5 & - & 2250 \\
des côtes & - & $(5,58)$ & - & $(8,21)$ & - & $(8,49)$ \\
TOTAUX & 72 & 34 & 3919,5 & 1858,5 & 85480 & 26491 \\
& $(100 \%)$ & $(100 \%)$ & $(100 \%)$ & $(100 \%)$ & $(100 \%)$ & $(100 \%)$ \\
\hline
\end{tabular}

* Il s'agit de deux marchands des côtes et d'un négociant de Montréal.

Sources : 81 actes de vente en 1784 et 37 actes en 1787 relatifs à des terres rurales des seigneuries de Saint-Sulpice, Lachenaie, Lavaltrie et Repentigny.

dans le sècteur foncier. La spéculation est impossible à cause de l'abondance des terres disponibles. De même, dans la seconde moitié du $18 \mathrm{e}$ siècle, les marchands ne possèdent aucun intérêt à s'impliquer directement dans la production de céréales destinées à la vente. Le blé et les autres grains achetés des paysans suffisent alors amplement aux demandes locales et extérieures, souvent instables.

Tout en adoptant diverses formes - achat de part de succession, échange avec ou sans soulte, donation à rente viagère et autres - les transactions foncières des marchands villageois de l'Assomption n'occupent qu'une faible place dans leurs pratiques économiques. Ainsi malgré sa longue carrière (1759-1792) et sa fortune évaluée à 83000 livres au moment de son décès ${ }^{43}, G$. Leroux n'achète, en tout ou en partie, que huit terres et quatre lopins ruraux. Ces achats de propriété, le plus souvent suivis d'une revente, recouvrent à peine une superficie de 300 arpents Pour sa part, Jean-Baptiste Durocher est plus actif dans le domaine foncier. Il acquiert et revend, entre 1752 et 1777, vingt-sept

\footnotetext{
43 ANQM, minute J. Papineau, inventaire après décès, 22 octobre 1794, no 2189.
} 
terres ou lopins de terre situés dans la région de l'Assomption. Néanmoins, ce marchand fait figure d'exception puisque la majorité des commerçants du bourg ont une action foncière limitée entre 1748 et 1792. Les revenus rentiers assurés par les fermes ou les profits procurés par la vente des tenures rurales ne justifient pas d'ailleurs, au 18e siècle, de la part de la communauté marchande de l'Assomption, l'investissement de sommes importantes dans le domaine foncier plutôt que dans les secteurs commercial et financier.

\section{CONCLUSION}

Le bourg de l'Assomption abrite, dans la seconde moitié du $18 \mathrm{e}$ siècle, une importante communauté marchande. Celle-ci étend son influence sur une vaste zone rurale située sur la rive nord du fleuve Saint-Laurent. Les marchands villageois concentrent principalement leurs actions dans le commerce de détail, des grains, du bois et des fourrures. Ces relations d'échange tissent des liens de dépendance financière entre les marchands, devenus créanciers, et leurs débiteurs, les paysans de la région. D'autre part, l'action marchande demeure marginale au niveau foncier qui ne constitue qu'un moyen parmi d'autres pour récupérer des créances douteuses.

Du pouvoir économique détenu par les marchands découle leur influence sociale. Ils occupent le premier rang au sein de la société villageoise et assument la presque totalité des charges civiles. Ils demeurent toutefois absents des postes paroissiaux. Cependant l'essor de l'activité marchande, au village de l'Assomption au 18e siècle, demeure totalement tributaire de la vitalité de l'économie et de l'expansion démographique dans la zone rurale environnante. Ces facteurs, présents dans les décennies 1770 et 1780 , contribuent fortement au développement du bourg de l'Assomption et assurent, du même coup, la formation et la consolidation de sa communauté marchande.

L'intervention de cette dernière dans les secteurs commercial et financier, dans la région de l'Assomption, supplante largement celle des négociants de Montréal, des marchands des côtes et des gros paysans. La prospérité de la communauté marchande villageoise repose d'ailleurs sur la place privilégiée qu'elle occupe dans la dynamique des échanges entre la ville et la campagne. 